\title{
Computational Study of Fluid Flow Inside a Concentric Reducer
}

\author{
N.Subaschandar \\ Department of Mathematics and Computational Sciences, BIUST, Palapye, Botswana
}

\begin{abstract}
Scale growth in pipe fittings is a major problem for an alumina refinery. Investigation into the scale growth mechanism at an alumina refinery found almost $60 \%$ more scale growth in concentric reducer when compared with the connecting straight pipe sections for similar flow conditions. The current study is an attempt to characterise the fluid flow inside a concentric reducer, especially streamwise and crossflow fluctuations using computational fluid dynamics methodology. The present study shows that streamwise component of instantaneous velocity fluctuation decreased and cross component of the instantaneous velocity fluctuation increased inside the concentric reducer. Present Computational results have shown good agreement with the experimental data.
\end{abstract}

\section{Introduction}

In alumina industry, scale growth in pipe fittings is a major problem for an alumina refinery. Investigation into the scale growth mechanism at an alumina refinery found almost $60 \%$ more scale growth in concentric reducer when compared with the connecting straight pipe sections for similar flow conditions[1]. In the present research an attempt is made to study the behaviour of streamwise and crossstream components of instantaneous fluctuating velocity inside the concentric reducer. A fully developed turbulent flow through an axisymmetric contraction producing constant strain rate was studied in the work[2]. They found that the streamwise component of fluictuating velocity is decreased and there was a similar increase in cross flow components in such a way that total turbulent kinetic energy remains constant in the initial section of the contraction. The streamwise component almost vanishes in the later sectFion of the contraction[2]. Recently, extensive measurements, mainly focusing on the behaviour of fluctuating velocity components as the flow goes through the reducer, have been carried out and presented in[1]. It was reported a decrease in the streamwise component of fluctuating velocity and an increase in the crossflow components of fluctuating velocity as the flow goes through the reducer[1]. Flow through contractions have been studied in[3-6]. Also axisymmetric flow through an expansioncontraction duct was studied in[7]. The present computational work studies the fluid flow through the concentric reducer, mainly focusing on the behaviour of fluctuating velocity components.

\section{Computational model}

The concentric reducer studied here is shown in the Figure 1. Computational domain extends on both side of the reducer by a distance of 4 diameters lengths. The water is the flow medium and flow is turbulent. Fluid is treated as incompressible fluid and, because of symmetry, the flow is treated as axisymmetric thus reducing the grid size and computational time. A uniform speed of $0.27 \mathrm{~m} / \mathrm{s}$ is considered at the inlet plane. Reynolds number based on the uniform speed of water and upstream diameter of the reducer is about 27,000. Pressure outlet condition was used at the exit plane. K- $\varepsilon$ turbulence model was used to model turbulence for all computations. Computations were carried out using the commercially available CFD software SOLIDWORKS. The computational domain had 112000 cells inside the reducer and this grid size was reached after carrying out grid independence study. Streamwise and crossflow fluctuating velocity components were computed at several streamwise stations inside the concentric reducer, as shown in Figure 2, to compare present computational results with experimental results[1].

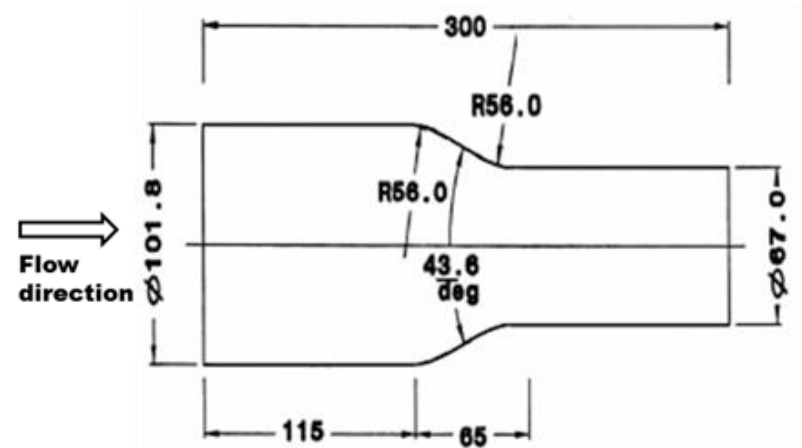

Figure 1 Sketch of the reducer geometry(All dimensions are in $\mathrm{mm})$. 


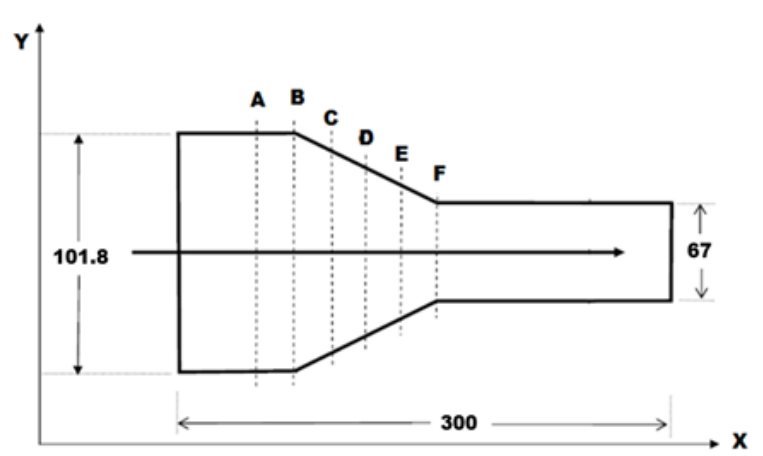

Figure 2 Streamwise locations at which the comparisons are made. $\mathrm{AB}=\mathrm{BC}=\mathrm{CD}=\mathrm{DE}=\mathrm{EF}=8$. (All dimensions are in $\mathrm{mm}$ ).

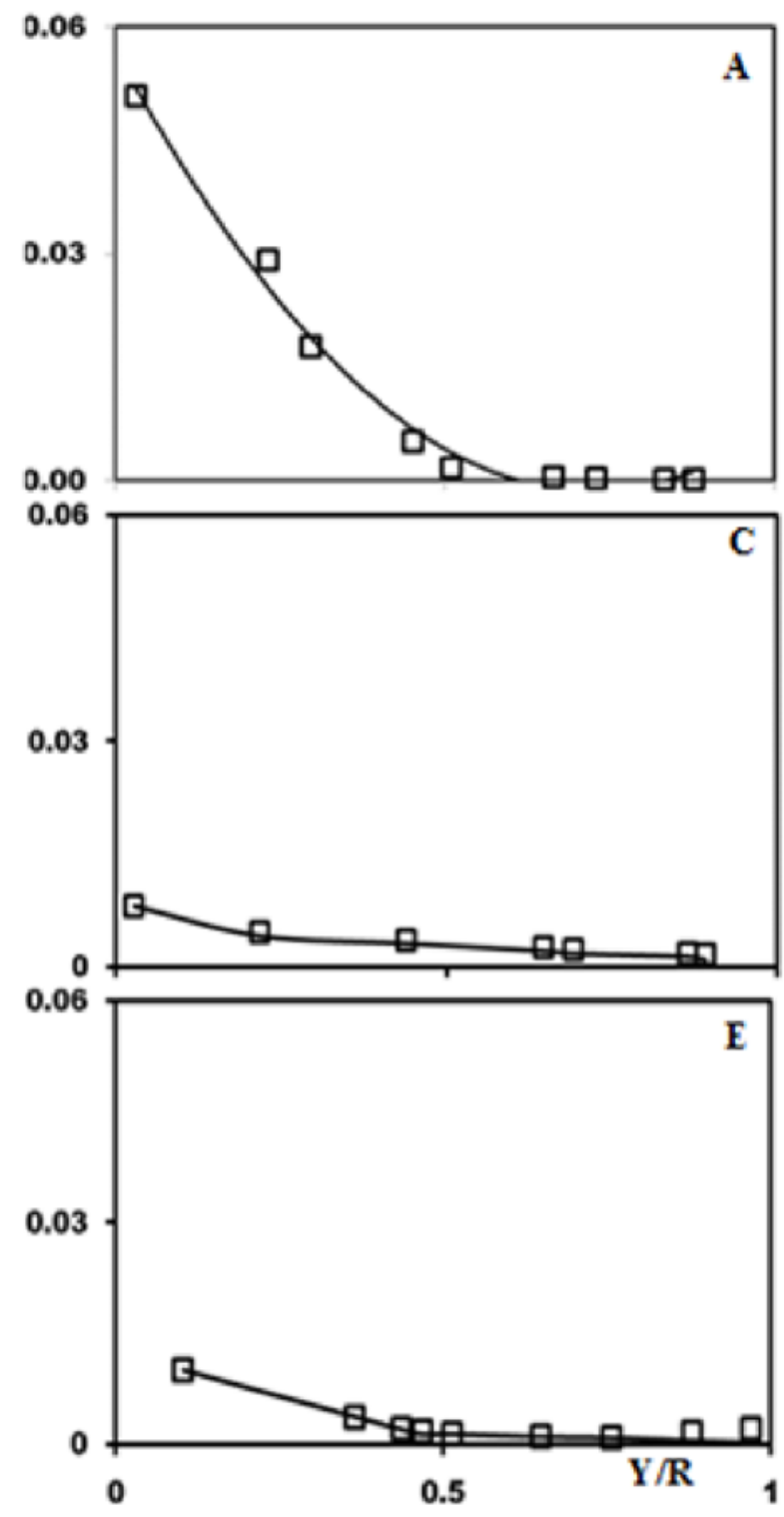

\section{Results and Discussion}

Figure 3 shows the comparison between computed streamwise component of velocity fluctuations and experimental data[1] at various streamwise locations inside the concentric reducer. It can be seen that the agreement between the computed and experimental results is reasonably good.
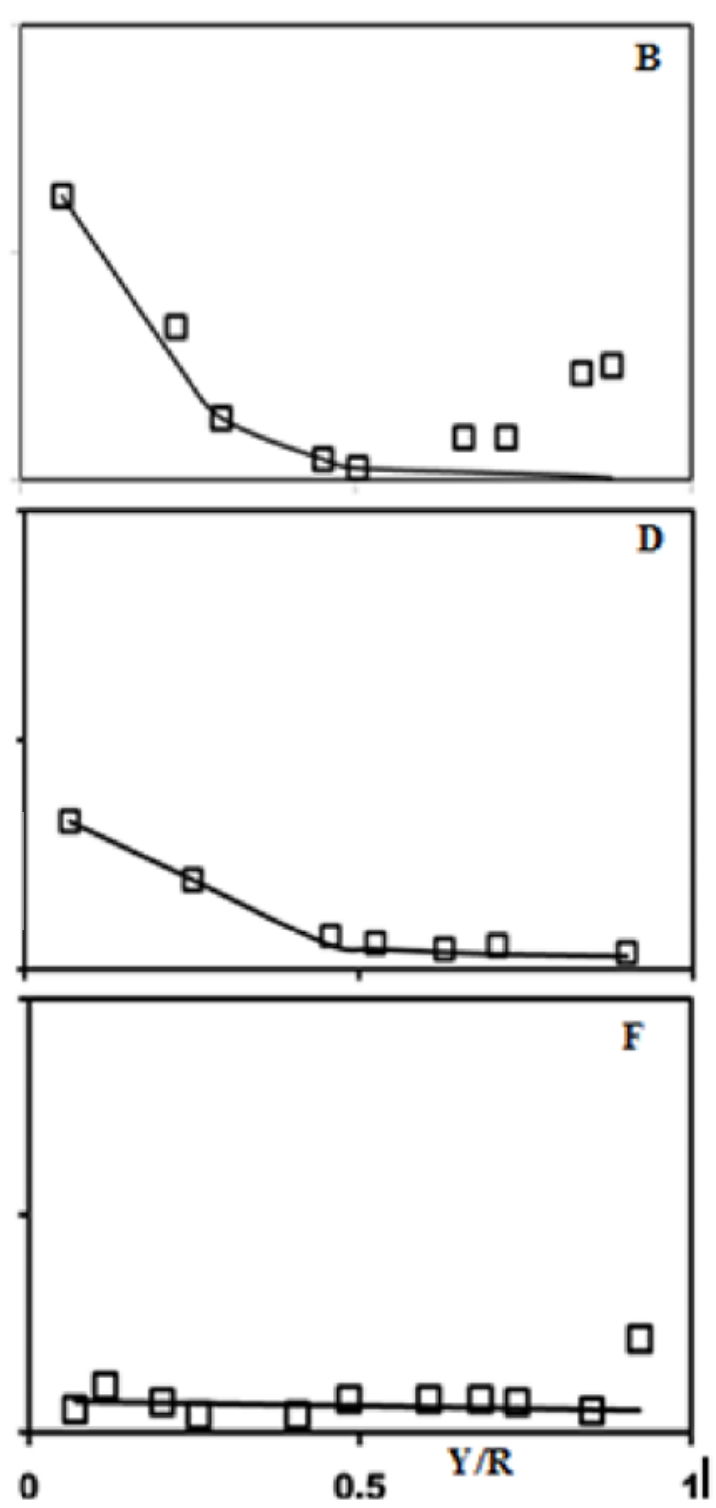

Figure 3 Variation of streamwise component of velocity fluctuation(u) through different sections.

\section{Vertical axis $-\left(\mathrm{u}^{2} / \mathrm{U}^{2}\right)$}

In the Figure 3B, the agreement between the computed result and experimental data is not good in later part of the graph; computations deviate from the experimental data as normal distance is increased. Figure 4 shows the comparison of computed results with that of the experimental data[1]. It can be seen from this figure that the agreement is not good, eventhough the computational results follow the trend of the experimental data. The experimental data has lot of scatter, which can be seen in the Figure 4, that makes comparison between computed results and experimental data very difficult. Figure 5 shows the comparison of computed results with that of 
the experimental data[1]. It can be seen from this figure, that even though there is a large scatter in the experimental data the computations follow the trend of the experimental results. We can see that the computations capture the small gradual decrease in the streamwise component of fluctuating velocity and the rise and fall of cross stream fluctuating velocity component along the streamwise direction at a location $\mathrm{Y} / \mathrm{R}=0.2$, which is near the wall. It is suspected that one reason for the reduction in the streamwise and crossstream components of fluctuating velocity could be the increase in the fluid velocity inside the reducer.
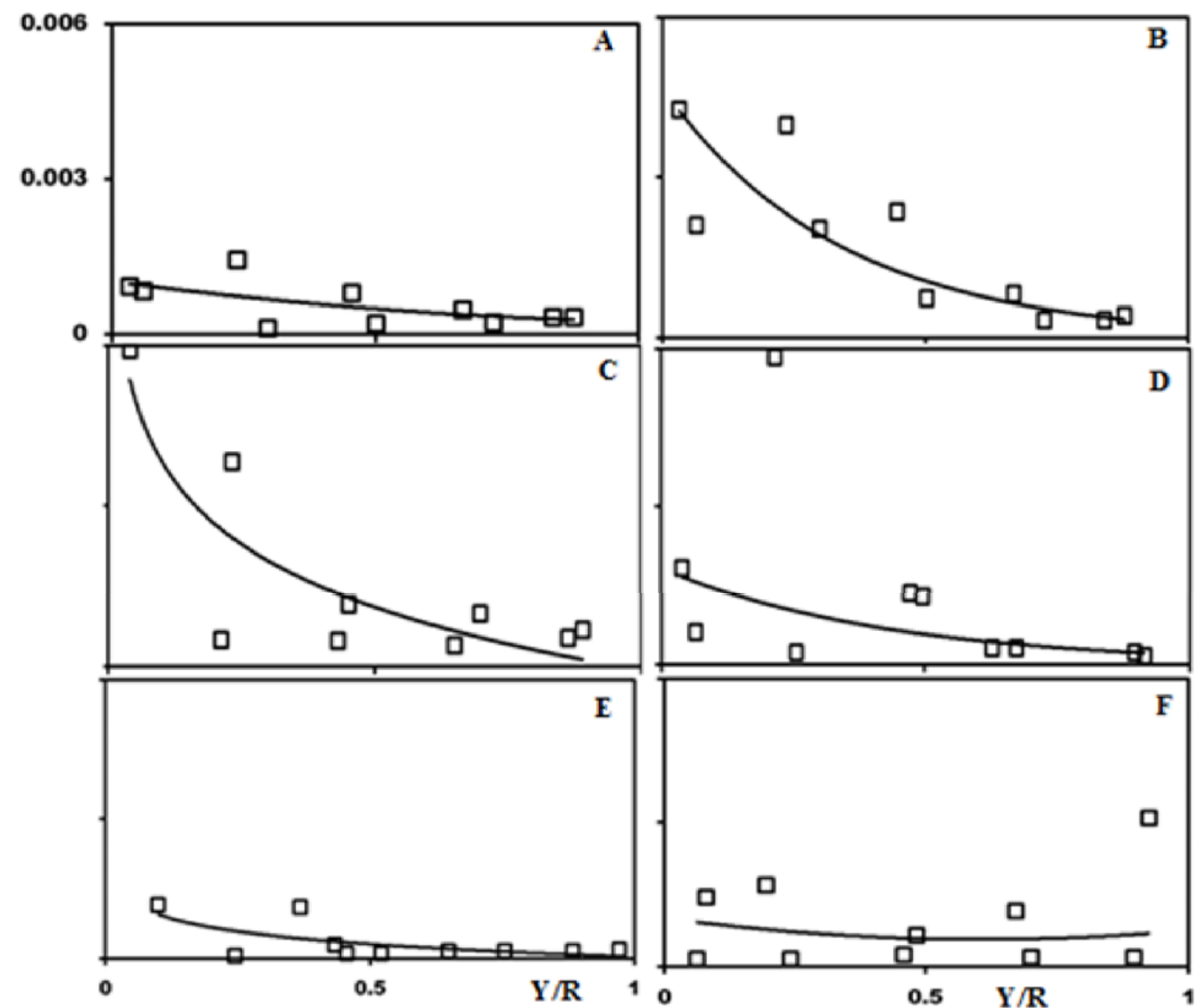

Figure 4 Variation of cross stream component of velocity fluctuation(v) through various sections. Vertical axis $-\left(\mathrm{v}^{2} / \mathrm{U}^{2}\right)$ 


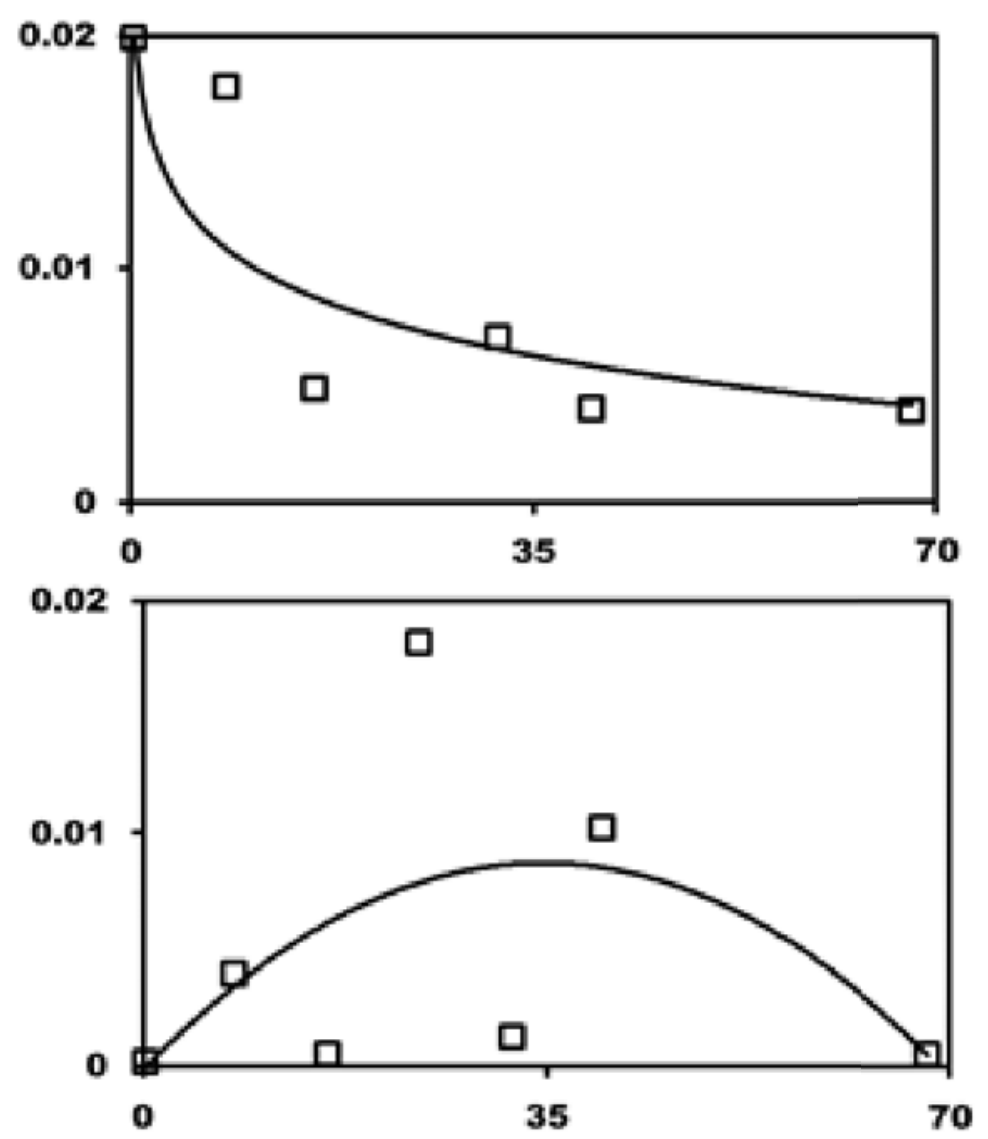

Figure 5 Variation streamwise and cross stream fluctuating velocity components near wall Vertical axis for $(A):\left(\mathrm{u}^{2} / \mathrm{U}^{2}\right)$; For $(B):\left(\mathrm{v}^{2} / \mathrm{U}^{2}\right)$

Fluid flow inside a concentric reducer is studied computationally. Computed results compare reasonably well with the experimental data. Computed results show that the streamwise component of fluctuating velocity decreases with normal distance. The computations do not predict the behaviour of crossstream fluctuating velocity component, but the trend is captured. The computations predict the variation of streamwise component of fluctuating velocity along the axial distance at a location close to the wall reasonably well. But the cross stream component of velocity fluctuation is not predicted satisfactorily. It is suspected that the increase in mean velocity in the reducer could be a reason for the decrease in the streamwise intensity.

\section{Acknowledgements}

Author acknowledges the support received from the Department of Mathematics and Computational Sciences and BIUST authorities in carrying out this research.

\section{References}

1. T. Rashid, Master's thesis, Dept. of Mech. Engg., Central Queensland University, QLD, (2005).

2. L. E. Tobergsen and P. Krogstad, Proceedings of the $13^{\text {th }}$ Australasian Fluid Mech. Conf., Monash University, Melbourne, 1, 378 (1998).
3. E. M. Kim, R. A. Brown, and R. C. Armstrong, J. of Non-Newtonian Fluid Mech., 13, 341, (1983).

4. J. P. Rothstein and G. H. McKinley, J. of NonNewtonian Fluid Mech., 86, 61 (1999).

5. J. P. Rothstein and G. H. McKinley, J. of NonNewtonian Fluid Mech., 98, 33 (2001)

6. P. Das, M. M. K Khan, M. G. Rasul, and S C. Saha, Proc. of 11th Int. Conf. on Heat transfer, Fluid Mechhermodynamics, 289 (2012).

7. M. Tachibana, N. Kawabata, and M. Sakamoto, Nihon Reororji Gakkaishi, 15, 210 (1987) 